\title{
GOSSYPIBOMA - A CASE REPORT
}

\section{INTRODUCTION}

Gossypiboma, the word is derived from latin word "gossipum" means cotton \& "Kiswahilbona", a place of concealment or retained surgical gauze. The condition is ubiquitous, iatrogenic avoidable complication with ethical \& forensic implication. They are retained more commonly than suspected $\&$ cause serious diagnostic problem and require secondary surgical procedures.

\section{CASE REPORT}

DMB 35 years, para III presented in a district hospital of Nepal with lower abdominal pain and a swelling of gradual onset since 5 months. Her menstruation was regular and last child birth was 10 years back. There was no urinary or bowel complaints or loss of appetite. She was advised an ultrasound examination which revealed ovarian cyst on right side. She underwent laparotomy and cyst was removed. She developed high grade fever with chills on third postoperative day with slight abdominal distension and lung infection. She was put on antibiotics and the symptoms gradually subsided and she was discharged on eight day after removal of stitches.

Twenty days after surgery she again developed distension of abdomen for which she consulted same hospital. The doctor noticed a swelling in lower abdomen aspirated pus from swelling and referred her to BPKIHS hospital, Dharan, Nepal.

When seen in BPKIHS emergency she was diagnosed as a case of parietal abscess and was admitted. On examination she looked sick but vitals were normal. Abdominal examination revealed a mass below the umblicus in the incision area partly cystic, tender and parietal in location. Pelvic examination revealed a normal size mobile uterus and normal adenexa. The abdominal mass was higher up and could not be reached

\section{Khanna S, Pokhrel H*, Basnet N, Chuni N}

on vaginal examination. On ultrasonography this mass was suspected to be a tetrad left from previous surgery. Foul smelling green pus drained through an spontaneous opening through which swab could be seen. Pus was sent for culture and sensitivity and exploratory laparotomy was done. Same opening was extended on incision line. On cutting the skin a sponge was found lying just underneath the incision extending from infraraumblical to pubic region. Sponge was attached to the surrounding rectus muscle by flimsy adhesions. On the posterior aspect of the sponge, omentum had sealed the peritoneal cavity (fig.1) and it was lying superficial to omentum with pus all around. Sponge was removed completely in one piece (fig.2). No attempt was done to enter abdominal cavity since omentum was adherent. A corrugated drain was put and skin was closed over the drain. One unit of blood was transfused.

Drain was removed on second day and she was put on injection Chloramphenicol, Amikacin and Metronidazole according to culture and sensitivity report which had grown Proteus. Patient recovered well and was discharged on tenth day.

Fig. 1

Fig.1 : Hole draining pus with tetrad underneath.

* B.P. Koirala Institute of Health Sciences, Dharan, Nepal.

Address for correspondence : Dr. Shail Khanna

Department of Obstetrics \& Gynaecology

B.P. Koirala Institute of Health Sciences, Dharan, Nepal. 


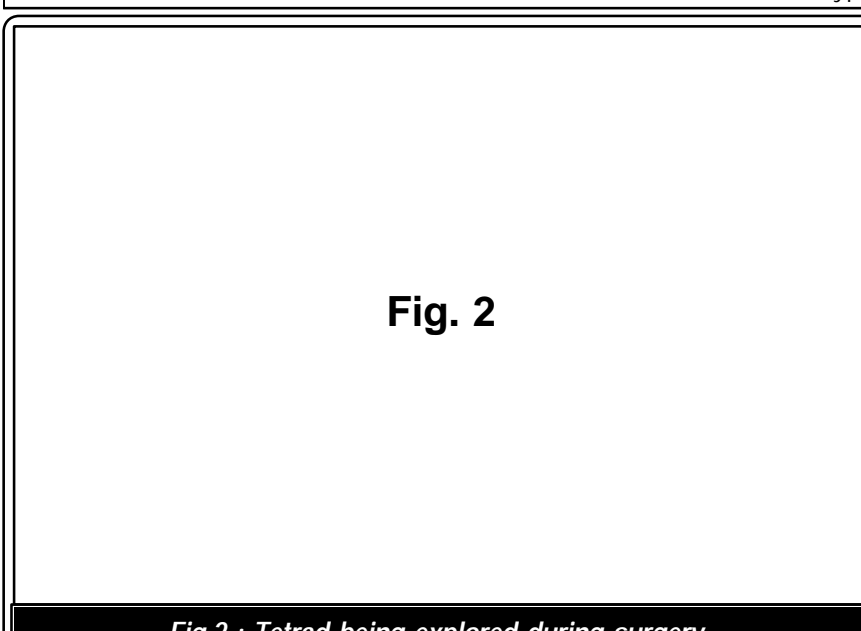

\section{Fig.2 : Tetrad being explored during surgery.}

\section{DISCUSSION}

Cases of retained sponge have been reported from both developed and developing countries, diagnosed in post operative period to as long as 40 years after surgery. ${ }^{1}$ This shows that it is a on going surgical problem. Interesting thing about it is, variable presentation which causes dilemma in diagnosis. A review of literature showed that it can present from a case of unexplained abdominal complaints, ${ }^{2}$ to mass simulating intestinal tuberculosis, ${ }^{3}$ Hepatic cyst, ${ }^{4}$ perinepheric abscess, ${ }^{5}$ Primary ovarian tumor, ${ }^{6}$ Recurrent epithelial ovarian cancer, ${ }^{7}$ Primary bowel tumour, ${ }^{8}$ intra abdominal cyst, ${ }^{9}$ Pancreatic \& renal foreign body granuloma, ${ }^{10}$ Intestinal obstruction \& tumor mass ${ }^{11}$ intestinal fistula. ${ }^{12}$ Presentation depends on sites of operation as well simultaneous occurance of foreign body. Foreign body granuloma away from primary surgical field is possible. Gauze forgotten after, it has infiltrated bladder wall, simulating invasive bladder tumours has been reported. ${ }^{13}$ Gossypiboma, migration of retained surgical gauze \& transurethral protrusion is also found in literature. ${ }^{14}$

A retained surgical sponge can cause either an aseptic reaction without significant symptoms or an exudalive reaction which results in early but nonspecific symptoms. Diagnosis can be confirmed by radiography which shows heterogeneous intra or extraluminous mass often containing air or calcification. Ultrasound CT scan \& MRI have variable appearance - sponge form pattern due to trapped air, complex mass of low or high density sometimes seen with high density rim or capsule. Radiopaque marker in sponge may help but is not to reliable. Management is expeditious removal by laparotomy or laparoscopy.

More important is prevention of such incidences. Even though counting sponges is a tedious task it should nonetheless be performed with utmost attention. Although the presence of radio-opaque wire in sponges is helpful in locating these, it does not prevent surgical sponges from being forgotten. All sponges should have tails \& count put on board before operation $\&$ checked after surgery. Lastly all the members in an operation team should work meticulously to prevent its occurrence.

\section{CONCLUSION}

History of fever and pain in postoperative period which is unexplained or a history of previous operation with palpable abdominal mass should always make a surgeon suspicious of retained sponge.

\section{REFERENCE}

1. KatoKSuzuki KandSai J et a .Acase of paravesi cal fore gnbody presenti ng as bl adder tunour. Urol I nt 2000; 65(4): 224- 25.

2. Schel hass E, MasboonwS. A ret ai ned Gauze as a cause of unexpl ai ned abdonnnal conpl ai nts Ned ti j dschr gencested 2002, 146 (6); 245- 46.

3. Gupta MM Bandi sh MF, Kunar A; Ret ai ned surgi cal sponge si nol ati gi ntesti nal tubercul osi sl ndj . Castoenter ol ogy, p97, 16 (2): 68- 69.

4. Moral as M Perezr, Pi eon G Retai ned surgi cal sponge si nol ati ng a hepat i c cyst. Rev Esp D g, 1994, 85 (6): 488- 49.

5. Bhatt M Rangopal K S. J R Gossi pi bona presenting as peri nephri c abscess surg Edi nb 1977, 42 (4): 277- 78.

6. GaHMi nCk, Yu Cpet al oxi di zedcel I uosesurgi cal granu ona nini cki nga pri nary ovari an tunour.I nt J gynaecol path 2002; 21 (4); 422- 23.

7. Ding $X$, Lang $J$, Shen $K$, Forgott en sponge presenti ng as recurrent epi the $\mathrm{i}$ al ovari an cancer. Zhonghua Funchan Ke Za Zhe 1999; 34 (1): 30- 32.

8. Zhar Ap, Agarwal A Seed J T. Gossypi bonæ revi si ted, present ati on as pri nary bovel t unour, J R col I surg Edi nb (1998); 43 (6:4127- 8).

9. Mbyl es $\mathrm{H} \mathrm{H}$ nes $\mathrm{Q}$, MCFadder DWGossypi bonæ of abdonen Arch Surg, 1996; B1 (5): 566- 68.

10. Kuo XT, Włng CK, W DK, Li u GC et al . I magi ng feat ures of si nol taneous occur ance of renal and pancreati c for ei gn body granul ona duet o chroni cal I y ret ai ned gauze. (P99) Kaohsi ung J. Ned Sci 1999; 15 (2): 104- 09.

11. SerraJ , Mati as - Gui al X, Cal abui g R et al .: Surgi cal gauze pseudot unour, Aneri can J. Surg. 1988, 155 (2): 235- 37.

12. Laconbe C D A i ncourt A, Ler at F, GMugnol E What i s i t? Abdonnnal forei gn body and snal I i ntesti nefi st u a;J Radi d 2000; 81(6): 64142.

13. Kyri akon GKal ahas N Kyroundi A Extravesi cl eforei gn body present i ng as bl adder t unour. Urol I nt 2000; 65(4): 244- 25.

14. J i n TY, Change CK, W DK, Li u GCet al . Cossypi bona ningrati on of retai ned sur gi cal gauze \&spont aneous transurethral protrusi on B.J.K. I nd 1999, 84(7) 879-80. 